\title{
Repensar la salud desde una Academia crítica y comprometida. Vida, acumulación y emancipación*
}

\section{Rethinking Health from a Critical and Committed Academy: Life, Accumulation and Emancipation}

\section{Repensar a Saúde desde uma academia crítica e comprometida. Vida, acumulação e emancipação}

Fecha de recepción: 11-04-14 Fecha de aceptación: 08-05-14 Disponible en línea: 01-07-14 doi: 10.11144/Javeriana.rgyps13-27.rsda

Cómo citar este artículo:

Abadía-Barrero C, Melo-Moreno M. Repensar la salud desde una Academia crítica y comprometida. Vida, acumulación y emancipación. Rev. Gerenc. Polít. Salud. 2014; 13(27): 41-57. http://dx.doi. org/10.11144/Javeriana.rgyps13-27.rsda

Artículo de reflexión

* Odontólogo, doctor en Antropología Médica, profesor Asistente del Departamento de Antropología y del Instituto de Derechos Humanos de la Universidad de Connecticut. Investigador del Centro de Estudios Sociales de la Universidad Nacional de Colombia. Correo electrónico: cesar_abadia@post.harvard.edu, cesar.abadia@uconn. edu. Correspondencia: César Ernesto Abadía Barrero. Department of Anthropology. University of Connecticut 354 Mansfield Road. Storrs, Connecticut 06269-1176. USA.

*** Antropólogo, magíster en Estudios Culturales, candidato a doctor en Antropología e investigador del Centro de Estudios Sociales de la Universidad Nacional de Colombia. Correo electrónico: marcoalejandromelomoreno@gmail.com 


\section{Resumen}

Se plantea la necesidad de cuestionar la hegemonía epistemológica, teórica y política de categorías como salud-enfermedad y bienestar, para proponer una perspectiva analítica diferente. Esta implica centrarse en la comprensión de las relaciones entre la praxis de los agentes sociales, la estructuración del régimen local de acumulación capitalista y el sufrimiento subjetivo que producen los diferentes sistemas de dominación y desigualdad social. Se presenta, en primer lugar, una reflexión sobre algunos elementos de teoría crítica que permiten contestar los marcos analíticos hegemónicos sobre las diversas dimensiones de las políticas de la vida y la salud, seguida por algunas alternativas para su comprensión. Posteriormente, se expone una aproximación a las problemáticas sociales claves que comprenden el desplazamiento analítico propuesto. Finalizando, se sintetiza la relevancia de una epistemología crítica del "cuidado de la vida" que logre contribuir, acompañar y potenciar luchas emancipatorias que enfrentan las expresiones violentas de distintos órdenes de dominación.

Palabras clave: capitalismo; principios morales; ciencias sociales; medicina social; atención a la salud

\section{Abstract}

We bring up the need to question the epistemological, theoretical and political hegemony of categories such as health-illness and well-being, to propose a different analytical perspective. This implies focusing on the understanding of the relationships between the praxis of social agents, the structuring of the local capitalist accumulation regime, and the subjective suffering produced by the different social inequality and domination systems. Firstly, we present a reflection on several critical theory elements that lead to an answer on the analytical hegemonic frameworks on the different dimensions of life and health policies, followed by several alternatives for its understanding. Afterwards, we present an approach to the key social problems that cover the proposed analytic shift. Finally, we summarize the relevance of a critical epistemology of the "care for life" that manages to contribute, accompany and strengthen the liberating struggles, faced with the violent expressions of different orders of domination.

Keywords: capitalism; moral principles; social sciences; social medicine; attention to health

\section{Resumo}

Coloca-se aqui a necessidade de questionar a hegemonia epistemológica, teórica e política de categorias como saúde-doença e bem-estar, para propor uma perspectiva analítica diferente. Esta implica se centrar na compreensão das relações entre a práxis dos agentes sociais, a estruturação do regímen local de acumulação capitalista e o sofrimento subjetivo que produzem os diferentes sistemas de dominação e desigualdade social. Apresenta-se, em primeiro lugar, uma reflexão sobre alguns elementos de teoria crítica que permitem contestar os quadros analíticos hegemónicos sobre as diversas dimensões das políticas da vida e a saúde, seguida por algumas alternativas para sua compreensão. Posteriormente, expõe-se aproximação às problemáticas sociais chaves que compreendem o deslocamento analítico proposto. Finalizando, sintetiza-se a relevância de uma epistemologia crítica do "cuidado da vida" que permita contribuir, acompanhar e potenciar lutas emancipatórias que enfrentam as expressões violentas de diferentes ordens de dominação.

Palavras-chave: capitalismo; princípios morais; ciências sociais; medicina social; atendimento à saúde 


\section{Introducción}

La antropología médica crítica y la medicina social abordan la salud de forma similar, como un campo de indagación en el que el bienestar, el enfermar y el curar/tratar se entienden como un conjunto de procesos continuos y contestados, que están configurados no solo por los entramados culturales, sino también por conflictos de poder históricos. Desde estas perspectivas, la salud y los ejercicios académicos que se desprenden de estas corrientes se entienden como un asunto político. Este texto se plantea como objetivo estimular el debate y la reflexión con respecto al centro político del accionar pedagógico e investigativo de las perspectivas críticas en salud, bajo la premisa de que ni la idea de salud ni la de bienestar nos indican hacia dónde podríamos orientar una perspectiva investigativa y política contrahegemónica. Se postula que dicha perspectiva tiene como núcleo analítico, ético y político la comprensión sobre cómo las distintas formas de dominación y explotación social generan, reactivan y transforman distintas desigualdades sociales y se inscriben en formas diferenciadas de existencia. Así, entendemos que las aproximaciones conceptuales y prácticas contenidas en las expresiones salud y bienestar emergen dentro de los campos de poder que definen la existencia humana y su comprensión crítica constituye un punto de entrada magnífico para abordar la relación entre vida, política y desigualdades sociales.

En esta propuesta, las respuestas sociales a los procesos de enfermar y curar/atender/ sanar, o preservar/fomentar/mejorar la salud o el bienestar, visibles tanto en el accionar individual como en el institucional y en las políticas públicas, hablan de las formas como los colectivos enfrentan y redefinen constantemente tanto lo viviente como la vida misma. Por viviente entendemos aquello que modula históricamente la existencia de los grupos humanos, siguiendo el sentido foucaultiano de biopoder. Como vida en sí misma, entendemos las modalidades de valoración diferenciada de distintas formas de existencia tanto singulares (sujetos) como colectivas (agrupaciones de diverso orden). Esto significa, en otras palabras, la comprensión de las jerarquías sociales de valor moral con las cuales se divide y se diferencia la existencia humana. Entendemos el valor moral no como un principio individual, ahistórico e incontestable de la buena voluntad del accionar humano, sino como una de las formas privilegiadas para acceder al dominio ideológico, en tanto su expresión es notable en las formas de vivir la vida propia, de valorar la vida de los grupos humanos y de discutir y actuar en el plano de las políticas públicas nacionales y mundiales.

Para entender a profundidad la moralidad vemos útil la articulación de dos planteamientos: el primero, que entiende la moralidad como la expresión de procesos subjetivos (inconscientes, emocionales o racionales) que llevan a las personas a actuar de forma aceptable en el mundo, en los que la valoración de lo justo o del deber ser se entiende como un aspecto que se moldea y se redefine constantemente, tanto en trasegares individuales como en transformaciones sociales profundas (1-3). El segundo enfatiza que el ejercicio del poder siempre implica la valoración diferenciada sobre la vida, es decir, una moralidad histórica en juego que termina desplegándose tanto en las políticas como en las experiencias vitales $(1,4,5)$.

En últimas, el ejercicio intelectual que proponemos consiste en ligar los procesos históricos que marcan experiencias de desigualdad, es decir, el dominio de unos grupos poblaciones sobre otros - principalmente bajo la hegemonía modernidad/patriarcado/ capitalismo (6) - con los procesos bajo los cuáles los mismos sujetos valoran la diferen- 
cia de la vida humana, como una forma de develar el accionar de las fuerzas ideológicas.

El artículo está organizado en cuatro apartados. En el primero se presentan las discusiones teóricas y se delimita el marco conceptual, con lo que se hace posible el desplazamiento de los marcos hegemónicos de comprensión sobre la salud y el bienestar hacia una comprensión crítica de las relaciones entre las experiencias vitales, las dinámicas del régimen de acumulación capitalista y la experiencia del sufrimiento como modalidad encarnada de sistemas de explotación y dominación social. El segundo apartado presenta una propuesta de reevaluación de dinámicas clásicas del pensamiento moderno en salud aplicando las herramientas teóricas planteadas. En el tercer apartado se discute el tipo de dinámicas sociales que, se considera, deben ser abordadas desde el desplazamiento epistemológico y político que se propone aquí para pensar críticamente las políticas de la vida. El cuarto apartado sintetiza las implicaciones que esta perspectiva tendría para una praxis emancipatoria sobre el dominio de lo que hegemónicamente se ha pensado e intervenido sobre el campo de la salud y el enfermar a nivel local.

\section{Discusiones teóricas y marco conceptual: teorías críticas y salud}

Breves elementos para una genealogía crítica del concepto de salud

A pesar de los importantes desarrollos que ha tenido el concepto de salud desde corrientes críticas como la medicina social, entendido en "positivo" como un proceso articulado al bienestar humano, queremos proponer una discusión a partir de la pregunta ¿qué implica que estos conceptos se comprendan dentro de la racionalidad moderna? No podemos olvidar que esta surge de la mano de trayectorias coloniales que afianzan un paradigma expansivo del capitalismo industrial a escala global. Este desarrollo, además, configura las relaciones de ciudadanía-Estado de modo que crea unos límites al desarrollo local y a las formas subalternas de construcción de solidaridad y de pensamiento alternativo, en relación con las prácticas de cuidado entre individuos, colectivos y organizaciones sociales complejas. En esa lógica, salud y bienestar pueden ser entendidos críticamente como elementos constitutivos de los mecanismos históricos del biopoder, que operan a través de dicotomías como salud/enfermedad, normalidad/anormalidad, o de la creación de segmentaciones espaciotemporales del mundo de la vida, en donde, por ejemplo, el proceso salud-enfermedad-atención es competencia del llamado sector salud o el proceso educativo de la institución escolar. Afirmamos que el problema de continuar pensando y desarrollando teorías que responden a rígidas divisiones disciplinares es el reforzamiento de una comprensión fragmentada de la experiencia vital del sujeto. Esto nos impide pensar, por ejemplo, cómo los procesos relacionados con la salud se viven en el espacio escolar y los procesos educativos en los escenarios de la salud.

\section{Perspectivas críticas latinoamericanas sobre el problema salud-enfermedad}

Esta crítica a la enfermedad y al ámbito institucional médico (la clínica) como centro de la lógica de funcionamiento (conceptual y práctico) de los ejercicios académicos en salud, ha sido bien establecida por la medicina social latinoamericana desde donde se propone desligar el pensamiento en salud de la lógica hospitalaria y salubrista para enmarcarlo como un ámbito de la vida misma. De lo que se trata es de contraponer un pensamiento sobre la salud y la vida al pensamiento hegemónico sobre la enfermedad (7), para 
entender que el deterioro de la vida implica principalmente asumir que la biología es un orden singular subsumido dentro del orden social (8). En esta lógica, el desarrollo de enfermedades ya no se entendería a partir de paradigmas causalistas "biosociales", sino como una resultante de dinámicas sociales como el trabajo, la producción y reproducción social, la relación ser-naturaleza y de procesos subjetivos como la praxis cotidiana y la formación de conciencia (8-11).

Edmundo Granda (12), por ejemplo, rescata que la medicina social latinoamericana se preocupa no solo por la enfermedad y la muerte, sino también por "reflexionar y entender la salud y la vida, sin descuidar los sujetos", y nos cuenta como "el vivir genera la salud y ésta no se da únicamente por descuento de la enfermedad".

Avanzando en una definición sobre salud, cita al plan de estudios de la Maestría de Salud Pública de la Universidad Nacional de Loja de 1997: "salud es una forma de vivir autónoma y solidaria, consustancial con la cultura humana, dependiente y condicionante de las relaciones que se establecen con la naturaleza, la sociedad y el Estado". A renglón seguido comenta: "Si en el diario deambular, las poblaciones producen su salud, entonces, la fuerza o poder fundamental para alcanzarla se encuentra en las poblaciones mismas y en su vida [...] Si se considera que la propia vida engendra salud, se requiere interpretar la vida mediante lógicas recursivas y aproximaciones ontológicas que privilegian al organismo como eje de conocimiento, el aprendizaje y la acción de cambio". Más que señalar posibles inconsistencias de esta propuesta trascendental después del análisis textual, lo que queremos resaltar es la dificultad que resulta de intentar definir la salud como un asunto estrechamente vinculado con la vida pero distinto de esta.

\section{De la salud a las políticas de la vida}

Si bien el significativo avance planteado con esta propuesta de "pensar en salud" (7) ha sido fundamental para una lucha contrahegemónica frente a la medicalización de las prácticas en salud, la reflexión que se pretende avanzar en este artículo es imaginar el tipo de desplazamiento político y académico que implicaría abandonar la categoría "salud" para afianzar una propuesta emancipatoria alrededor de la categoría "vida" o de una categoría más sociológica como "políticas de la vida y de lo viviente". Bajo esta mirada, se propone que "la salud" debería dejar de ser pensada como sustantivo y convertirse en adjetivo calificativo de la vida, en el marco de las dinámicas de funcionamiento del capitalismo como modo de producción. Esto se ve mucho más claramente en propuestas como "deterioro de la vida" (13) y cuando se identifican y se ponen en discusión "modos de vida malsanos" en Ecuador, los cuales representan un retroceso del derecho a la vida fruto de la aceleración de la acumulación de capital a escala global y las prácticas específicas de despojo en el periodo neoliberal en ese país (14).

Si consideramos que la salud es más que bienestar, y si entendemos el bienestar como la mayor potencialidad humana, entonces estamos hablando de todas las condiciones materiales y espirituales de la existencia de la especie humana y su interdependencia con las demás especies y las dinámicas propias de la biósfera. En ese marco, lo que proponemos es enfocar nuestra perspectiva crítica de investigación y emancipación en la confrontación de los desarrollos históricos de las políticas de ordenamiento social, es decir, en las estructuras normativas de la sociedad, en el tipo de vida humana que se valora como útil en dicho ordenamiento y en las condiciones de "felicidad" que dichos sujetos deben encarnar. 


\section{La moralidad política de la vida}

Esta propuesta se puede sintetizar como "una moralidad política de la vida", inspirada en los desarrollos teóricos de Fassin (4). Con este concepto se quiere significar cómo el cuidado de la vida está configurado por los mecanismos de desigualdad, explotación y sujeción propios de la dominación de clase/ raza/género y orden colonial contemporáneo, que se expresan a través de una organización jerárquica del cuidado de la vida. De acuerdo con lo anterior, las posibilidades y las restricciones para el buen vivir se organizan de acuerdo a dichos procesos históricos de diferenciación y jerarquización que se encarnan en la experiencia de las personas, en la medida en que se consolida este esquema jerárquico de valoración/valorización de la vida. Este produce unos mecanismos de juzgamiento de la conducta de las personas donde el ajuste a diferentes moralidades en salud, como el autocuidado y la adopción de estilos de vida saludables, es entendido ideológicamente como el cumplimiento de meros "actos de voluntad individual". En la lógica del mérito y la voluntad se ocultan las desigualdades objetivas y sus distintos procesos de materialización en sujetos particulares que determinan la adopción de dichas prácticas, aquellas que surgen de la interacción entre sistemas duraderos de desigualdades sociales como la clase, el género, la raza y el orden colonial. Estos mecanismos operan, en el orden práctico y simbólico, como marcadores de diferencia que, con variaciones según el momento histórico, distinguen entre las vidas que tienen la posibilidad "de ser preservadas incondicionalmente" o que "merecen considerarse" y aquellas que son calificadas y administradas como "banales".

Queremos destacar, al contrario de las categorías de pensamiento burgués, cómo esas 46 diversas moralidades tienen unas condiciones sociales de posibilidad, materiales y sim- bólicas que constituyen el fundamento sobre el cual es posible ejercer el tipo de agencia que la racionalidad liberal piensa como "natural". Un ejemplo de las determinaciones objetivas de estas moralidades puede verse cuando analizamos las condiciones de vida de las poblaciones que carecen de sistemas básicos de saneamiento ( $\sin$ acueducto o alcantarillado) y viven situaciones de inseguridad alimentaria permanente. Por ejemplo, la investigación de Fabián Ardila (15), dentro de nuestro grupo de investigación, muestra cómo una situación de inseguridad alimentaria permanente, una de cuyas manifestaciones visibles es la desnutrición infantil, es "paliada" a través de intervenciones sociales y de atención clínica que buscan la superación de los ciclos más críticos de la "desnutrición proteico-calórica", pero que niegan o carecen de propuestas frente a las causas de la precariedad de las condiciones materiales de vida de las poblaciones subalternas, toda vez que dichas intervenciones se basan en limitar el problema de la desnutrición a suministrar alimentos (gratuitos o subsidiados) o a la prescripción de dietas que incluyen múltiples alimentos que son de imposible adquisición.

Así, no debemos pensar la valoración de la vida, en su régimen contemporáneo, en términos absolutos sino como una construcción específica que se da en momentos históricos concretos. La jerarquización moral de las vidas humanas es un mecanismo estructurante de las sociedades contemporáneas, donde las vidas que ocupan las posiciones dominadas en el espacio social, las clases subalternas, son administradas y gestionadas a través de la lógica clínica o de políticas de mejoramiento de la calidad de vida. Estas constituyen una forma de entender la experiencia vital de las poblaciones de clases subalternas, a través de la superación sucesiva y fragmentada de eventos "patológicos" o de la adopción de estilos de vida saludables (como una 
buena dieta en este caso), individualizando los procesos sociales que jerarquizan y modelan las vidas humanas. Estas vidas se valoran en cuanto se incorporan a través de intervenciones episódicas que reproducen esa condición dominada y excluida de las vidas de las poblaciones subalternas y cuya valoración, bajo la figura de indicadores que suben o bajan, termina sirviendo como barómetro ideológico de las mismas políticas de dominación.

\section{Propuestas de reevaluación}

Repensar la dicotomía entre salud y enfermedad

Esta (di) visión moderna de la vida basada en la dicotomía entre salud y enfermedad o terapéutica clínica y políticas/programas, y que en sus desarrollos actuales se presenta como gradientes que permanecen en los conceptos "salud, bienestar y calidad de vida", es parte del dispositivo de poder que pretende restituir a grupos y sujetos sociales una condición aceptable de vida fisio-biológica para la socialidad, civilidad y productividad a partir de la intervención clínica o de programas que se sustentan en resultados específicos y medibles en el corto plazo.

¿Cómo replantear entonces estudios y acciones que se interesan en "mejorar" las condiciones de vida de las personas? Frente a los postulados que evalúan sistemas de protección social en salud a través de, por ejemplo, la calidad de atención del sistema, se propone que una forma de comprenderlo es como un "circuito de reciclaje" que hace parte de la lógica de valorización capitalista contemporánea. Por un lado, intenta con su intervención en las etapas más visibles y definitivas del malestar, que son en la mayoría de los casos la culminación de un proceso vital de larga duración determinado por desigualdades sociales y su encarnación en sujetos específicos, restituir las mínimas condiciones que permiten la (potencial) reproducción individual de la fuerza de trabajo como mercancía. Por el otro, sirve como regulador funcional del ejército de trabajadores y trabajadoras de reserva por cuanto controla su flujo de entradas y salidas, al restituir, expulsar o excluir temporalmente a contingentes de la fuerza de trabajo. Como ejemplo de lo anterior encontramos cómo en situaciones de deterioro de las condiciones de salud de algún miembro de la familia, sus miembros deben emplearse o encontrar actividades generadoras de ingresos, percibiendo remuneraciones que están por debajo de los estándares mínimos de vida, para poder pagar los múltiples gastos de bolsillo que en los casos más favorables incluyen los gastos de movilización burocráticos que el conjunto interminable de autorizaciones implica.

Bajo esta mirada es posible redefinir el cuidado que familiares "prestan" al sistema de salud. Por ejemplo, María Teresa Buitrago (16) demuestra que la necesidad de cuidado de los y las personas con malestares severos en sus vidas, requiere la autoexpulsión de las cuidadoras "informales" del mercado laboral, con todos los efectos deletéreos que esta situación implica para los sujetos en el capitalismo, tales como el deterioro de la identidad personal, la "dependencia" y la restricción objetiva en sus condiciones materiales de vida.

Siguiendo esta perspectiva, el desplazamiento epistemológico implica, entonces, no investigar, por ejemplo, los significados de la enfermedad renal o la calidad de vida de una persona que vive con enfermedad renal, sino preguntarnos qué lugar ocupa en esta jerarquía moral la valoración de las vidas que transitan con este proceso. Una expresión de esto es entender la valoración que los sistemas de salud hacen de estas vidas y los 
acuerdos morales que subyacen, los cuales se presentan como hegemónicos con respecto a las necesidades de vidas con enfermedades específicas. En el caso de la reforma de mercado del sistema de salud colombiano, entendido no como una política pública en sí, sino como expresión de la hegemonía neoliberal que enfatiza un valor y una valoración específica sobre la relación vida-enfermedad-costos de atención, la enfermedad renal crónica y grave solo es (o era, ya que las estrategias de valorización son contestadas y cambian) atendida después de cinco años de pagar la póliza de aseguramiento. Así, se exige el pago anticipado del procedimiento, con su respectivo margen de ganancia, antes que la necesaria atención clínica. Efectivamente, la calidad de vida está comprometida, pero esto se debe a la valoración moral que se da dentro de la estructura capitalista a estas vidas, en las que estas aparecen como costos "marginales" o "excepcionales" y por tanto experimentan una existencia condicionada a la lógica de acumulación.

Si bien hemos utilizado el caso de la enfermedad crónica, tal análisis es posible realizarlo en todos los casos en los cuales se consolida la lógica de costo-efectividad o de la "finitud" de los recursos económicos disponibles como principios que racionalizan las posibilidades de atención integral.

\section{Desnaturalizando el bienestar}

En cuanto al bienestar, ilustremos cómo una postura acrítica pierde de vista la construcción de la moralidad neoliberal sobre la vida, por medio de los procesos de renovación urbana que, supuestamente, mejoran las potencialidades humanas de todos los habitantes de la ciudad. En el caso del Plan Centro en Bogotá se puede observar que la revitalización urbana es representada, en términos del bienestar colectivo, como el mejoramiento generalizado de las condiciones de vida de toda la población urbana a partir de la mejora de la circulación, la generación de empleo, la estética urbana, etc. Sin embargo, la valoración diferencial de la vida se expresa si se entiende cómo este proyecto implica un proceso de expulsión de las poblaciones de clases subalternas, donde confluyen distintas dinámicas violentas para despojarlas tanto de sus viviendas ${ }^{1}$ como de la ventaja espacial que representa habitar en estos barrios.

En los casos mencionados, la inseguridad alimentaria y su expresión física como desnutrición, el cuidado de familiares y la precarización económica por el afianzamiento de la expulsión de la fuerza de trabajo, la enfermedad renal y su expresión como problema de costos, acceso y calidad, y el Plan Centro de Bogotá como ejemplo de la destrucción de oportunidades de vida que es presentada, cínicamente, como una política de bienestar colectivo, se observa que la moralidad política de valoración/valorización de la vida obedece a los principios de mercado y a la estructuración de nuevas configuraciones de clase, en las cuales la vida es confrontada porque solo adquieren tratamientos o bienestar aquellos 'merecedores', ${ }^{2}$ y ese merecimiento (valoración de la vida) se da en términos de los diversos tipos de capitales que se osten-

$\overline{1}$ Un ejemplo de esta lógica se puede observar en el proceso de 'compra' de las viviendas que ocupan la zona futura de Ciudad Salud. Aun cuando se otorguen viviendas en el mismo sector, la mayoría de los propietarios verían reducido su espacio de vivienda, teniendo en cuenta que sus casas son de varios pisos y tendrían que reducirlo a las dimensiones prescritas para la vivienda de interés social (en el mejor de los casos de alrededor de $50 \mathrm{~m}^{2}$ ).

2 También se puede ser 'merecedor' de asignaciones a través de la lógica neoliberal de focalización de la pobreza, ya que personas que demuestren no tener recursos pueden acceder a tratamientos. Para el caso de los habitantes del centro, su pertenencia histórica a estos espacios los hace 'merecedores' a subsidios de vivienda que les generan malestar por la reducción de su espacio habitacional y, adicionalmente, los endeuda y les reduce sus ingresos derivados de la renta parcial de sus propiedades. 
tan (económico, simbólico, social, etc.), los cuales se articulan con la nueva concepción de ciudadanía neoliberal, en la cual cada individuo debe gestionar su propia atención y es responsable último de su calidad de vida (17). Así, cuando el capital avanza y ve en la salud no solo una esfera de control social, sino nuevas posibilidades de acumulación, empezamos a presenciar que mientras que las vidas de las clases dominantes encuentran nuevos mecanismos para mantener o mejorar su existencia, las de las clases subalternas se desvalorizan (y devalúan) cada vez más, expulsándolas de las redes laborales o llevándolas a la informalidad, o cuando sus lugares de vivienda y sus cuerpos son deteriorados o debilitados, no son rehabilitados, o se consideran poco productivos.

\section{Dinámicas sociales relevantes}

Para profundizar en esta propuesta crítica se desarrollan tres dinámicas sociales que permiten ver mejor los alcances y limitaciones de plantear estudios críticos sobre la valoración moral de la vida en el capitalismo, como propuesta alternativa a aquellos estudios que se basan en las ideas de salud, bienestar o calidad de vida. Estas dinámicas son: 1) la experiencia del sujeto entendida como encarnación intersubjetiva de la historia; 2) el proceso de acumulación de capital en salud y las dinámicas burguesas de control social; y 3) dinámicas de emancipación.

\section{La experiencia del sujeto entendida como encarnación intersubjetiva de la historia}

La modernidad y su desarrollo en una filosofía política liberal nos han llevado a pensar en los sujetos como unidades analíticas independientes, libres y autónomas. Estos son los principios básicos que sustentan la gubernamentalidad moderna, en donde la suma de las transacciones entre los ciuda- danos, como portadores y defensores de sus intereses individuales, constituiría el dominio de la política y el gobierno.

Así, por ejemplo, para "tratar" a un enfermo con diabetes, nuestra unidad de intervención será el sujeto que la padece, y para saber qué piensa de sus cuidados, indagaremos a esta persona y estableceremos unas pautas de comportamiento para que se haga partícipe de ellos. El conocimiento médico reside en forma absoluta en el individuo aislado como unidad ontológica. Es fácil ver los límites de este ejercicio, en el cual se sustenta la clínica, ya que se asume que la "unidad clínica-analítica" corresponde a un sujeto ahistórico y neutro. Por tanto, siguiendo tradiciones ya clásicas en antropología médica y en medicina social, es claro que la relación curativa médico-paciente que se plantea como un ejercicio entre dos sujetos modernos, corresponde a una práctica intersubjetiva construida históricamente en donde el mundo de la vida es reducido a sus símbolos orgánicos (signos, síntomas, diagnóstico y tratamiento). Asimismo, la traducción de la condición humana a términos biomédicos resulta en un conocimiento parcializado, en el cual lo "social" es visto como un conjunto de epifenómenos poco significativos para entender la enfermedad (18). Aún más, al desconocer, minimizar y ayudar a desechar la influencia de la historia vital y de las condiciones de vida adversas en el malestar, se ha planteado que el ejercicio clínico termina transmitiendo mensajes ideológicos y de control social en donde la persona acepta el infortunio, desestima las necesarias transformaciones sociales para obtener bienestary aprende a aceptar las injusticias como parte de las dificultades de la vida, dificultades frente a las cuales solo se puede actuar a través de terapéuticas clínicas individuales (19).

La constitución dual del sujeto expresa, por un lado, la sedimentación de la serie de interacciones y relaciones recíprocas de poder 
que constituyen las trayectorias y situaciones particulares de los sujetos, lo que podríamos llamar subjetividad, y por el otro, la posición objetiva que estos ocupan dentro de los diferentes sistemas de dominación y explotación social. Asimismo, el sujeto está demarcado por las instituciones de la gubernamentalidad liberal-burguesa, que enmarcan los límites normativos de su acción y con el tiempo consolidan la moralidad de la vida, es decir, las formas sociales de la conciencia (ideológicas) y su normalización en el orden moral (la valoración diferencial de la vida).

Así, al ser producto de relaciones sociales, cada sujeto es entendible en cuanto que experiencia de vida, en la cual encarna y es interpelado por todas las demás experiencias humanas de los sujetos que, en una u otra escala, lo afectan. Por tanto, entendemos que cada sujeto es "muchos sujetos" y que muchos sujetos se expresan y son visibles en cada uno. Planteamos, entonces, que una posible salida a esta forma moderna de ver el sujeto es no ver sujetos, sino intersubjetividades. Esto implica entender que cada interacción humana es un encuentro entre tramas intersubjetivas que son estructuradas por dinámicas históricas particulares (20).

Esta breve crítica de la racionalidad moderna nos permite entender que la libertad (que sustenta gran parte de las propuestas, políticas y programas de cuidados en salud) no solo es una construcción liberal-burguesa, sino que es al mismo tiempo una tecnología de gobierno, un conjunto de dispositivos que controlan el dominio y el flujo de poder del campo de las intersubjetividades, y también una construcción ideológica (21). Aquí es necesario reconocer cómo la libertad individual, principio de la individualidad burguesa, se convierte en una condición abstracta, vacía y sin sustancia, puesto que la explotación y la alienación, propias de la hegemonía moderno-capitalista-patriarcal-colonial, no permiten la realización de las libertades humanas ni el florecimiento de las condiciones para la creación de subjetividades plenas.

Uno de los procesos más interesantes es entender la libertad individual como práctica ideológica en salud, ya que no hay proceso más social, interdependiente, vulnerable y anclado a desigualdades de poder que el enfermar y buscar mecanismos de sanación. Por ejemplo, la escogencia adecuada de un plan de salud, o inclusive pensar en el uso de mecanismos jurídicos para la resolución exitosa de los problemas de salud, depende del uso estratégico de los distintos tipos de capitales (económicos, culturales y sociales que Bourdieu identifica). Estos capitales, que se relacionan con desigualdades sociales históricas, también se vinculan estrechamente con las barreras económicas, geográficas y administrativas derivadas de los procesos de acumulación de capital en salud que, paradójicamente, se justifican en el ordenamiento jurídico que regula el derecho a la salud en el país $(20,22)$.

Además de lo anterior, la racionalidad que sustenta la libre escogencia es ilógica, si se tiene en cuenta la imposibilidad de obtener información suficiente. Por ejemplo, si requiero decidir entre dos neurocirujanos para que me traten de urgencia un accidente cerebrovascular, tendría que conocer sus trayectorias de formación, de práctica clínica, y conocer con suficiencia los aspectos técnicos de la patología y la terapéutica para decidir cuál es la mejor opción dentro del mercado. Frente a esto, lo que las personas consideren como mejor opción y su relación con mejor atención tuvo que sufrir un cambio. Si con respecto a mi accidente cerebrovascular mi idea de calidad de atención se relaciona con el mejor resultado posible, toda opción de mercado debería ofrecérmela y, por tanto, la escogencia no tendría sentido. Para revaluar esta idea se necesita replantear la mirada 
sobre la calidad de la atención en salud, que actualmente se da en términos de productos industriales que miden la satisfacción del usuario y la ligan estándares supuestamente iguales con libre escogencia.

Otro ejemplo de lo anterior se expresa en la individualización de procesos sociales a través de mecanismos como la meritocracia y su traducción en salud, y los estilos de vida saludables, que niegan la forma como los regímenes de dominación social determinan las posibilidades de acción de sujetos particulares. Otra estrategia en ese sentido es la culpabilización de las víctimas como forma de violencia simbólica que las hace responsables por su propia condición de subordinación y por sus diferentes experiencias de sufrimiento y exclusión.

Entender la enfermedad escindida en componentes biológicos y sociales o bio-psicosociales se sustenta en otra de las principales construcciones de la política liberal- burguesa: la separación analítica entre individuo y sociedad, así como la idea de que la sociedad resulta de la suma de los individuos. Si consideramos que no se puede pensar en una separación, ni siquiera analítica, entre sociedad e individuo debemos entender, como postuló Bourdieu en innumerables ocasiones $(23,24)$, que la sociedad se encarna en el individuo, pero que sería falso argumentar que el individuo se encarna en la sociedad.

Al plantear que la sociedad se encarna en el sujeto, queremos señalar que los sujetos son constituidos por relaciones sociales materiales, es decir, por esas dinámicas de clase que articulan de una manera particular las relaciones de explotación, control y apropiación diferencial del trabajo vivo y objetivado. En el capitalismo, la relación médico-paciente es una expresión metonímica de todas las determinaciones sociales que constituyen a estos sujetos. En el caso de las relaciones de clase, la interacción entre el profesional de la salud y el paciente está regulada por la defensa de los intereses de las clases dominantes por parte de los profesionales del campo biomédico y de las relaciones de sujeción que estos ejercen sobre sus pacientes (25).

Debe quedar claro que esta situación no es una forma de práctica deliberada de las y los profesionales en el campo biomédico, sino que responde a estas lógicas estructurales de organización de la sociedad en su conjunto. Es decir, las posibilidades de una práctica humanista por parte del profesional de la salud están determinadas por el carácter del trabajo clínico, que le imprime una lógica capitalista a la prestación del servicio, asî como por las tecnologías de explotación basadas en la optimización del uso del tiempo y en la eficiencia como el mayor número de casos-pacientes atendidos durante una jornada de trabajo. Todo esto sin contar con los procesos sociales propios del complejo médico-industrial y otros grandes intereses corporativos (por ejemplo de las compañías farmacéuticas, de seguros y los hospitales privados) que no pueden prescindir del trabajo médico, pero recrean constantemente formas para explotarlo y dominarlo.

\section{El proceso de acumulación de capital y las dinámicas burguesas de control social}

Para esta sección tomamos como centro analítico la reforma de mercado al sistema de salud en Colombia con la Ley 100 de 1993. Entendemos que esta es una experiencia particular en donde han sido más evidentes los intereses de clase, pero pensamos que los ejes analíticos críticos son aplicables a otras esferas de valorización del capital en salud en otros países. La propuesta general de esta sección es que la mercantilización de la salud se basa en dos falacias: 1) la competencia de mercado y 2) el paradigma liberal aplicado a la salud. 
Todos estamos de acuerdo en que el sistema de salud en Colombia desde las reformas neoliberales de los noventa funciona bajo principios de mercado. Pero itodos los que decimos esto seguimos un pensamiento anclado en teorías críticas? En la práctica, todos los sectores involucrados están de acuerdo en que el sistema necesita mayores mecanismos de control y algunos enfatizan en que los problemas estructurales del sistema requieren una reforma profunda. Sin embargo, no todos piensan que el sistema de salud refleje las nuevas dinámicas de acumulación de la fase actual del capitalismo. Estas distintas posturas marcan diferencias conceptuales, metodológicas, éticas, políticas y epistemológicas en la integración de las perspectivas críticas al estudio y la transformación de las realidades que enfrentamos con el sistema de salud actual. Su situación financiera se muestra como una coyuntura propicia para entender cómo su lógica ni siquiera se corresponde a una racionalidad económica neoclásica —el máximo de rendimientos con la menor cantidad de recursos disponibles - sino que obedece a intereses corporativos de clase orientados hacia la maximización de la rentabilidad en el sector y la consolidación del ordenamiento jurídico que la hace posible. Esto implica, del mismo modo, que las reformas formuladas desde el Estado al sistema, o bien buscan limitar los recursos sociales disponibles para la restitución de condiciones mínimas de salud de las poblaciones (expresados en las limitaciones al derecho de tutela con la Ley 1473 de julio de 2011 y las redefiniciones constantes de los 'paquetes' de beneficios incluidos o excluidos en las pólizas), o bien garantizar el adecuado flujo de transferencias de recursos fiscales hacia procesos privados de acumulación (los recobros del Fosyga también consolidados con la nueva ley o la amenaza de transformar el carácter público de los recursos del sistema una vez entran a las arcas de las aseguradoras).
A contramano de los clichés académicos corrientes, pensar y proponer mecanismos de transformación del campo de la salud a través de una perspectiva crítica de inspiración marxista es muy distinto a pensar la salud bajo una visión "economicista". Lo que queremos poner de relieve aquí es que la fase neoliberal del desarrollo del capitalismo está fundada en la subordinación de todas las esferas de la vida a su propia lógica de acumulación. Las dinámicas actuales del sistema de salud se corresponden con su subsunción real y formal a la esfera de valorización capitalista. Esto quiere decir que la salud es un núcleo de acumulación de capital en sí mismo (un sector donde se realiza el proceso de autovalorización del capital a través de los circuitos de producción e intercambio), en el que compiten varios sectores capitalistas por la maximización de las ganancias. Entre sus agentes más visibles encontramos el sector seguros, el complejo médico-industrial y las compañías farmacéuticas. Adicionalmente, la organización de la explotación del trabajo en este sector no solo ha incorporado creativamente las formas neoliberales de sobreexplotación, flexibilización y pauperización laboral, sino que ha logrado aplicar mecanismos de control administrativo propios de la financiarización a través de tecnologías administrativas, como el tiempo de consulta, o mediante el establecimiento de una nueva jerarquía en la que los gerentes de los hospitales, con una mirada rentista de la salud, subalternizan a los profesionales que prestan los servicios.

Es evidente que los principios de racionalización y control propios del régimen disciplinario industrial (la producción de plusvalor absoluto y relativo) siguen vigentes, a la par que se instauran nuevas formas de acumulación flexible, control financiero y salida de capitales del sector, mediante la instauración de márgenes de intermediación administrativa. En esta lógica económica, que siguiendo a 
Marx es una dinámica histórica propiamente social y política, es que podemos comprender a nivel micro y macro social muchas de las dimensiones de construcción práctica y discursiva de la salud a nivel global y local.

Así, las categorías de pensamiento y la división social del trabajo que organizan a la salud como campo, solo se pueden comprender si entendemos como están constituidas por las dinámicas históricas de producción capitalista, que revoluciona y, simultáneamente, preserva distintas configuraciones de las relaciones sociales y los principios de visión y división que constituyen la vida social (23, 24). Por esto, es necesaria una comprensión crítica de las racionalidades que están en el "corazón del sistema": realizar la crítica de las ideas fundamentales y los esquemas conceptuales que constituyen la formación ideológica actual en el campo de la salud.

\section{El papel del Estado en el régimen actual de acumulación capitalista en salud}

Perspectivas críticas han entendido que el sector de la salud es, históricamente, un campo de pugnas entre actores capitalistas o con deseos capitalistas. Sin embargo, algunos argumentarán que no podemos dejar de lado el papel regulador del Estado. Efectivamente, la acción e intervención del Estado es un mecanismo básico en las lógicas de acumulación del modo de producción capitalista. Podemos señalar, sin embargo, qué es lo que diferencia la propuesta que aquí se presenta de aquellas que fundamentan su praxis política en la omnipotencia del sujeto jurídico de la lógica burguesa y en la potencia cierta de la institucionalidad estatal para resolver los conflictos sociales. Es importante entender que esta última afirmación no tiene un carácter normativo, sino un carácter histórico. Durante el siglo xx, la acción del Estado en la regulación del ordenamiento social propio del capitalismo ha oscilado entre una función de estímulo y protección a los procesos de valorización y concentración del capital, y una lógica de funcionamiento que corresponde a una forma-Estado que se configura objetivamente a partir de las relaciones de fuerza entre clases (y otros sistemas de ordenamiento) que constituyen el conflicto social. Lo importante aquí es reconocer el conjunto de fuerzas que hacen imposible en el momento actual en Colombia un cambio en el régimen hegemónico, expresado en reformas sociales, a través de un cambio en el Estado.

Consideramos, entonces, que hay dos posibilidades de adscripción a corrientes de pensamiento 'crítico'. La primera la representan quienes entienden que estamos frente a un problema de limitación en los recursos que requiere la expansión del gasto social. En este marco, se comprende que es necesario negociar con el Estado por medio de los mecanismos liberales de participación para que nuestras demandas sean escuchadas, ya sea por medio de mesas de negociación y/o concertación, o con la formación de bloques que impulsen esta visión en el Congreso. Las respuestas a la crisis actual del capitalismo para este neokeynesianismo, con algunas posturas de la izquierda como sus principales aliados, confían en la ilusión "reformista" y "la progresividad de los derechos", que se asientan sobre la expansión y optimización de los mecanismos de regulación del capital y el papel del Estado en la preservación del orden social.

Desde una perspectiva crítica marxista, entendemos cómo dichas tentativas de regulación social no se corresponden con el momento histórico del desarrollo capitalista en Colombia; que necesita, por un lado, enfrentar la crisis social desencadenada por el descontento generalizado frente al sistema de salud, pero, por el otro, seguir utilizando la estructura del sistema para continuar con este nuevo ciclo de acumulación de capital. 
El gobierno colombiano, en cabeza del ministro de Salud, ha aceptado en más de una ocasión que la solución de la presente crisis en el sistema colombiano requeriría la transferencia de nuevos recursos fiscales, que no hacen parte de las transferencias corrientes en salud, para poder "sanear" las dificultades financieras de las aseguradoras en salud (conocidas en Colombia como EPS o empresas promotoras de salud o, según la nueva ley, gestores de servicios de salud). Estos mecanismos actuales de redistribución corresponden estrictamente a una socialización de la crisis. En otras palabras, estamos frente a una transferencia de parte del valor producido por el trabajo colectivo, representado en los recursos fiscales y parafiscales, hacia el capital concentrado en el sector de la salud. Por tanto, podemos afirmar que no hay señales objetivas que permitan guardar esperanza alguna en el retorno del viejo keynesianismo como una lógica que permita reconstituir la hegemonía de clase sobre líneas de compromiso sociopolítico con los intereses de las clases trabajadoras y subalternas.

En el sistema de salud colombiano actual se expresa claramente la imposible articulación de las luchas a través del orden político-normativo vigente, por lo menos en tres formas: 1) la protección de la rentabilidad del capital privado a expensas de la protección de la vida (porque en el caso colombiano inclusive la propia función mínima del Estado para proteger la vida depende de garantizar la rentabilidad a los sectores privados en salud, ante el ya casi definitivo desmantelamiento de la red hospitalaria pública); 2) la cooptación por parte de grupos mafiosos de los recursos y el control administrativo de la salud; y 3) la diferenciación política a nivel del poder legislativo por medio de intereses clientelistas de las élites regionales o de intereses particulares de las distintas fracciones del bloque de clases hegemónico. En este sentido 54 expresamos, por un lado, el tipo de Estado que tenemos en Colombia y su imbricación con las mafias y, por otro lado, las posturas aparentemente críticas que deslegitiman cualquier tipo de lucha o reivindicación que se dé por fuera de los marcos normativos. Así, Marx ya nos planteaba que debemos pensar que el Estado desempeña un papel fundamental en el mantenimiento y la ampliación de los privilegios de la clase dominante (26), los cuales se traducen en distintos intereses que varían históricamente según las dinámicas de acumulación particular, incluso con la especulación sobre futuras patologías (27), y las luchas que los contrarresten parcial o totalmente.

En últimas, en la actual "problemática de la salud" el papel del Estado sigue siendo la maximización de la ganancia y la minimización del conflicto social, a través de nuevas tecnologías de gestión como formas mínimas de protección de la vida (por ejemplo, la necesidad de acudir a mecanismos jurídicos para obtener acceso a servicios indispensables para la vida que pueden ser interpretados como mecanismos de dominación de clase, al crear la idea de que existe una lógica práctica en la dupla negación-protección del derecho) y de viejas tecnologías, muy utilizadas en Latinoamérica, como la criminalización de las resistencias. Es decir, no es posible hacer una reforma "exitosa" al sistema de salud (expresada en una cobertura universal, de calidad y eficiente) a través de los mecanismos jurídicos y/o de representación política en el legislativo, cuando el momento hegemónico actual no deja dudas de la dominancia de la protección de la acumulación del capital en el sector de la salud, a despecho de cualquier intento de establecer un sistema que implique un escenario de redistribución y democratización de los servicios de salud por vía del Estado.

\section{Dinámicas de emancipación}

Retomemos la discusión sobre salud como bienestar, o salud "en positivo", en la que se 
argumenta que estas perspectivas no logran desprenderse de formas de pensamiento colonial modernas, entendidas como biomedicina. Ya se planteó que una forma de descolonización implicaría hablar sobre la vida y no sobre la salud. Sin embargo, frente a este postulado, ¿cuál es el papel de las enfermedades en ese proceso de la vida? Se afirma que las enfermedades son parte de la vida y que salud-enfermedad no deben comprenderse como un par dicotómico. Así, planteamos que la enfermedad hace parte del continuo de la experiencia de los sujetos $\mathrm{y}$, por tanto, las respuestas a ellas deben verse como componentes integrales de las trayectorias vitales y sociales. Adicionalmente, una propuesta contrahegemónica tendría que revertir la hegemonía del conocimiento especializado sobre la enfermedad, para ponerla al servicio de las trayectorias vitales y sociales de los sujetos. De esta forma, se piensa que la acción terapéutica debe acompañar las lógicas sociales y no introducir discontinuidades al subordinar el proceso de vivir con y sin enfermedades a la lógica de la clínica o de los programas de salud pública. De acuerdo con esta propuesta, el conocimiento especializado debería dialogar con las lógicas del discurso de la experiencia y las dinámicas locales para diseñar conjuntamente formas de acompañar la vida, con y sin enfermedades, y reconocer sus alcances, pero también sus limitaciones frente a problemas que no puede solucionar y repensar su accionar debido a los múltiples problemas que nutre o introduce para las luchas emancipatorias.

Lo anterior no implica la desaparición del conocimiento ultraespecializado, si bien es claro que la reforma flexneriana ${ }^{3}$ introdujo

3 La reforma flexneriana se refiere al desarrollo científico y regulado de la práctica médica que siguió al informe presentado por Abraham Flexner en 1910 a la Asociación Médica de Estados Unidos. Tal énfasis científico de la educación médica transformó profundamente el modelo anterior preceptorial y conformó la lógica de la práctica médica escindida en especialidades. una idea equívoca de la medicina como ultraespecialidad clínica, en pugna con la salud comunitaria o atención primaria. Sin embargo, aún se reconoce necesaria la ultraespecialidad. En el ejemplo del accidente cerebrovascular es lógico que necesitamos neurocirujanos bien preparados que puedan atender tal evento, pero este conocimiento experto estaría en la jerarquía más baja de la praxis de necesidades. Es decir, no hay un predominio a priori del saber experto, sino que este aparece como uno más que contribuye a la resolución de los problemas sociales y cumple su papel cuando se necesita, pero no prima en determinar la salud poblacional.

En este apartado nos guiamos por el concepto de conciencia 'contradictoria' de Gramsci, de acuerdo con el cual todo reconocimiento radical de la diferencia implica también entender que esta se encuentra constituida por experiencias de desigualdad epistemológica. Así, las experiencias locales se estructuran en el doble juego entre la formación de la reproducción de la hegemonía y la praxis emancipatoria del trabajador que se adscribe a una epistemología crítica del cuidado de la vida. Esta debería, por un lado, contribuir, acompañar, potenciar la posibilidad de ruptura respecto a las experiencias, prácticas y conocimientos que están determinados por condiciones de subordinación y sujeción social, y por el otro, preservar y reproducir el saber y el hacer de las categorías subalternas que permita comunicarlas a otras experiencias locales y de articulación de las luchas globales. Así, el cuidado de la vida no se encarna necesariamente en el médico o profesional de la salud y no necesariamente está pensado como responsabilidad de un sujeto particular e identificable, sino que al reconocer que el sujeto histórico deviene de procesos de sujeción que a su vez incluyen herramientas de liberación, se piensa como un asunto de emancipación de clase para conseguir la constitución de una nueva he- 
gemonía que, necesariamente, es de carácter anticapitalista y poscolonial.

Lo que proponemos es entender que el problema del cuidado de la vida está imbricado en el conjunto de estructuras de dominación propias de las formaciones sociales en el capitalismo tardío (con sus nuevas expresiones de clase, género, raza y orden colonial), y que si bien el conocimiento técnico sobre el cuerpo enfermo es importante, lo es mucho más la comprensión y la acción política. Solo de esta forma, las construcciones locales del cuidado de la vida podrían atacar, diezmar y eliminar la desigualdad social y la marginalidad como el paso más importante para llegar a diferenciar, valorar y enriquecer la diversidad de la vida humana.

La reivindicación de las posturas críticas hoy, en cabeza del marxismo, no implica un retorno idílico a los tiempos del socialismo real ni un rechazo de las nuevas formas de movilización sociopolítica. Mucho menos implica desconocer las críticas a los regímenes totalitarios o revivir la falsa idea de que la utopía socialista es realizable bajo formas organizativas estatales liberales. La reivindicación del marxismo como propuesta teórica y práctica implica reconocer y trabajar con las transformaciones en las dinámicas del modo de producción capitalista (28-30), pero también actualizar su fuerza interpretativa, explicativa y emancipadora con y a través de otras teorías críticas sobre la desigualdad social, como algunas vertientes del feminismo, el poscolonialismo, el posestructuralismo, el antirracismo, etc. Sin embargo, nuestra postura crítica parte de reconocer cómo las contradicciones del modo de producción capitalista en su fase actual modelan, de una manera particular, todas las dinámicas y macroestructuras de dominación y explotación social. Lo anterior no parte del reclamo de una "primacía ontológica" de las relaciones sociales capitalistas, sino que reconoce el lu- gar central y determinante que estas ocupan en la constitución de las líneas de fuerza de las sociedades contemporáneas.

Así, todas las luchas emancipatorias encuentran en la transformación del orden capitalista el marco de realización de todas sus potencialidades y demandas, ya que proponemos que los límites de las formas de acción política orientadas sobre el eje de reconocimiento identitario residen en su alcance insuficiente para la formación de una nueva hegemonía transformadora de las relaciones sociales capitalistas.

\section{Referencias bibliográficas}

1. Fassin D. A Contribution to the Critique of Moral Reason. Anthropological Theory. 2011 Dec; 11 (4): 481-91.

2. Kleinman A. What Really Matters. Living a Moral Life amidst Uncertainty and Danger. Oxford: Oxford University Press; 2006.

3. Zigon J. Moral and Ethical Assemblages A Response to Fassin and Stoczkowski. Anthropological Theory. 2010 Mar; 10 (1-2): 3-15.

4. Fassin D. Entre las políticas de lo viviente y las políticas de la vida. Hacia una antropología de la salud. Revista Colombiana de Antropología. 2004; 40 (enero-diciembre): 283-318.

5. Fassin D. Another Politics of Life is Possible. Theory Culture Society. 2009; 26 (5): 44-60.

6. Grosfoguel R. Del final del sistema-mundo capitalista hacia un nuevo sistema-histórico alternativo: la utopística de Immanuel Wallerstein. Nómadas. 2006; 25 (octubre): 44-52.

7. Testa M. Pensar en salud. Buenos Aires: Lugar Editorial; 1993.

8. Breilh J. La determinación social de la salud como herramienta de transformación hacia una nueva salud pública (salud colectiva). Rev Fac Nac Salud Pública. 2013; 31 (supl 1): S13-S27.

9. Breilh J. Epidemiología crítica. Ciencia emancipadora e interculturalidad. Buenos Aires: Lugar Editorial; 2003.

10. Breilh J. Epidemiología: economía, medicina y política. Tercera edición. México, D.F: Distribuciones Fontamara; 1986.

11. Almeida-Filho Nd. La ciencia tímida. Ensayos de deconstrucción de la epidemiología. Buenos Aires: Lugar Editorial; 2000.

12. Granda E. ¿A qué llamamos salud colectiva, hoy? Revista Cubana de Salud Pública. 2004; 30 (2): 1-20.

13. Breilh J, Campaña A, Costales P, Granda E, Páez R, Yépez J. Deterioro de la vida: Un instrumento para 
análisis de prioridades regionales en lo social y la salud. Quito: Corporación Editora Nacional; 1990.

14. Breilh J, Tillería Y. Aceleración global y despojo en Ecuador. El retroceso del derecho a la salud en la era neoliberal. Quito: Universidad Andina Simón Bolívar, Abya Yala; 2009.

15. Ardila F. Ciudadanía alimentaria, hambre y malestares en Soacha, Cundinamarca. En Abadía Barrero CE, Góngora Sierra AL, Melo Moreno MA, Platarrueda Vanegas CP, editores. Salud, normalización y capitalismo en Colombia. Bogotá: Universidad Nacional de Colombia, Universidad del Rosario, Ediciones Desde Abajo; 2013. pp. 77-92.

16. Buitrago MT. "Uno se vuelve cuidador, como ustedes dicen, por obligación”. Las redes de poder en el oficio de cuidar a personas en situación de discapacidad. En Abadía Barrero CE, Góngora Sierra AL, Melo Moreno MA, Platarrueda Vanegas $\mathrm{CP}$, editores. Salud, normalización y capitalismo en Colombia. Bogotá: Universidad Nacional de Colombia, Universidad del Rosario, Ediciones Desde Abajo; 2013. pp. 77-92.

17. Horton S, Barker J. "Stains" on their Self-Discipline: Public Health, Hygiene, and the Disciplining of Undocumented Immigrant Parents in the Nation's Internal Borderlands. American Ethnologist. 2009; 36 (4): 784-98.

18. Kleinman A. Writing at the Margin. Discourses between Anthropology and Medicine. Berkeley, Los Angeles, Londres: University of California Press; 1995.

19. Waitzkin H. The Politics of Medical Encounters. How Patients and Doctors Deal with Social Pro- blems. New Haven y Londres: Yale University Press; 1991.

20. Abadía-Barrero C, Oviedo D. Intersubjetividades estructuradas: La salud en Colombia como dilema epistemológico y político para las ciencias sociales. Universitas Humanística. 2008; 66 (juliodiciembre): 57-82.

21. Žižek S. Violence: Six Sideways Reflections. Londres: Profile Books; 2008.

22. Abadía-Barrero CE, Oviedo D. Itinerarios burocráticos de la salud en Colombia: la burocracia neoliberal, su estado y la ciudadanía en salud. Revista Gerencia y Políticas en Salud. 2010; Sup 9 (18): 86-102.

23. Bourdieu P. El sentido práctico. Madrid: Taurus; 1991.

24. Bourdieu P. La distinción. Madrid: Taurus; 1999.

25. Waitzkin H. A Marxist View of Medical Care. Annals of Internal Medicine. 1978; 89: 264-78.

26. Jasso-Aguilar R, Waitzkin H. El Estado, las multinacionales y la medicina contemporánea. Palimpsestus. 2007/2008; 6: 69-82.

27. Sunder Rajan K. Biocapital. The Constitution of Postgenomic Life. Durham y Londres: Duke University Press; 2006.

28. Boron A. Por el necesario (y demorado) retorno al marxismo. En Boron A, editor. La teoría marxista hoy. Buenos Aires: Clacso; 2006. pp. 35-52.

29. Harvey D. Breve historia del neoliberalismo. Madrid: Ediciones Akal; 2007 [2005].

30. Kincheloe J, McLaren PL. Rethinking Critical Theory and Qualitative Research. En Denzin NK, Lincoln YS, editors. Handbook of Qualitative Research. Thousand Oaks: Sage; 2005. pp. 303-42. 